\title{
fondations superficielles sur sol amélioré par inclusions rigides verticales
}

\author{
amelioration of soils by vertical rigid piles, \\ for shallow foundation
}

\author{
O. COMBARIEU
}

Laboratoire régional des ponts et chaussées de Rouen*

Rev. Franç. Géotech. n० 53, pp. 33-44 (octobre 1990)

\section{Résumé}

Cette étude complète celle qui a été réalisée en 1986 sur l'amélioration, par des inclusions rigides, de sols supportant des remblais. Elle propose des méthodes relativement simples de dimensionnement de tels réseaux destinés à recevoir, par l'intermédiaire d'un matelas intercalaire, soit un radier souple, soit une semelle superficielle rigide.

Une comparaison avec les quelques cas publiés d'application sur ouvrage ou expérimentaux montre que ces méthodes sont applicables.

\footnotetext{
Abstract

This study complete the one carried out in 1986 on the amelioration of soft soils under embankments by vertical rigid piles. This paper set up relatively simple methods for design such rigid piles network, which is loaded through intermediate fill material, by flexible raft foundation or rigid footing.
} 
Cette étude est la poursuite logique de celle engagée en 1986, concernant la construction de remblais sur sol médiocre amélioré par des inclusions rigides verticales, qui s'est traduite par un article publié dans la Revue Française de Géotechnique (1988), et qui a donné lieu à une communication au Congrès International de Mécanique des Sols et des Travaux de Fondation de 1989 à Rio de Janeiro.

Le présent article a pour objet l'étude des possibilités de fondations superficielles sur des sols médiocres améliorés par le même procédé. On y propose des méthodes de dimensionnement d'un tel réseau que l'on confronte aux quelques rares cas concrets d'application réelle ou de recherche, publiés dans la littérature technique.

\section{PRINCIPES DE CONSTRUCTION}

Si, pour fonder un ouvrage à la surface du sol, que ce soit par l'intermédiaire d'une fondation de grande dimension (radier), ou d'une semelle rigide (de faible largeur), on choisit d'améliorer le sol par des inclusions rigides, on est tout logiquement conduit à prévoir et ménager, entre la base de la fondation et la tête des inclusions, une couche intercalaire de maté- riau. Cette obligation résulte souvent de plusieurs préoccupations simultanées ou non :

- la première réside dans le principe même de la technique qui est l'amélioration d'un sol lui conférant de nouvelles propriétés d'ensemble. Ainsi traité, ce sol est destiné à recevoir des ouvrages fondés " superficiellement $»$, sans liaison mécanique particulière autre que le simple contact traditionnel semelle-sol;

- la deuxième est liée au simple souci, dans le cas de sols franchement médiocres, de pouvoir évoluer et construire sur une plateforme qui soit circulable, et qui mette les têtes d'inclusions à l'abri des détériorations possibles ;

- enfin, la troisième peut être dictée par le simple encastrement minimal que nécessite la condition de mise hors gel du sol sous la fondation.

Ces différentes considérations conduisent aux schémas de principe suivants (fig. 1), en remarquant que le sol intercalaire est en règle générale un sol rapporté de bien meilleure qualité que le sol en place naturel; il en sera d'ailleurs obligatoirement ainsi dans le cas des radiers où ce * matelas * reconstitué remplit une fonction très importante de transmission de la charge vers les têtes d'inclusions. On peut imaginer cependant pour les semelles que ce soit le sol natu-

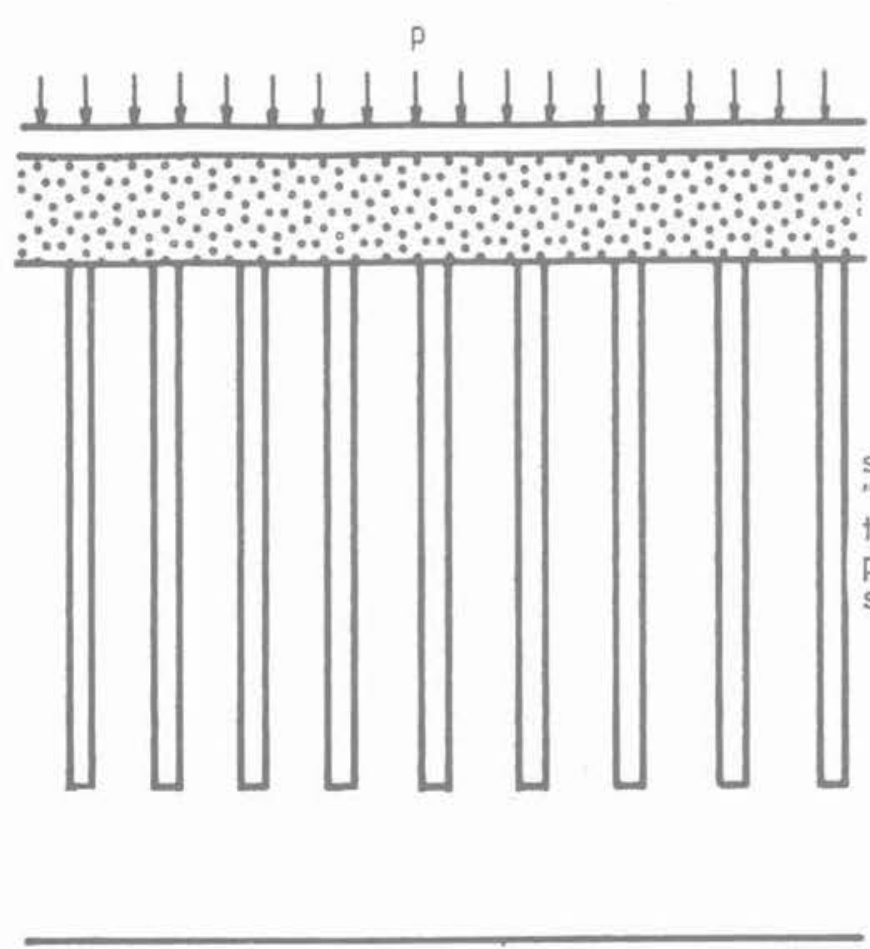

sol de bonne quallité

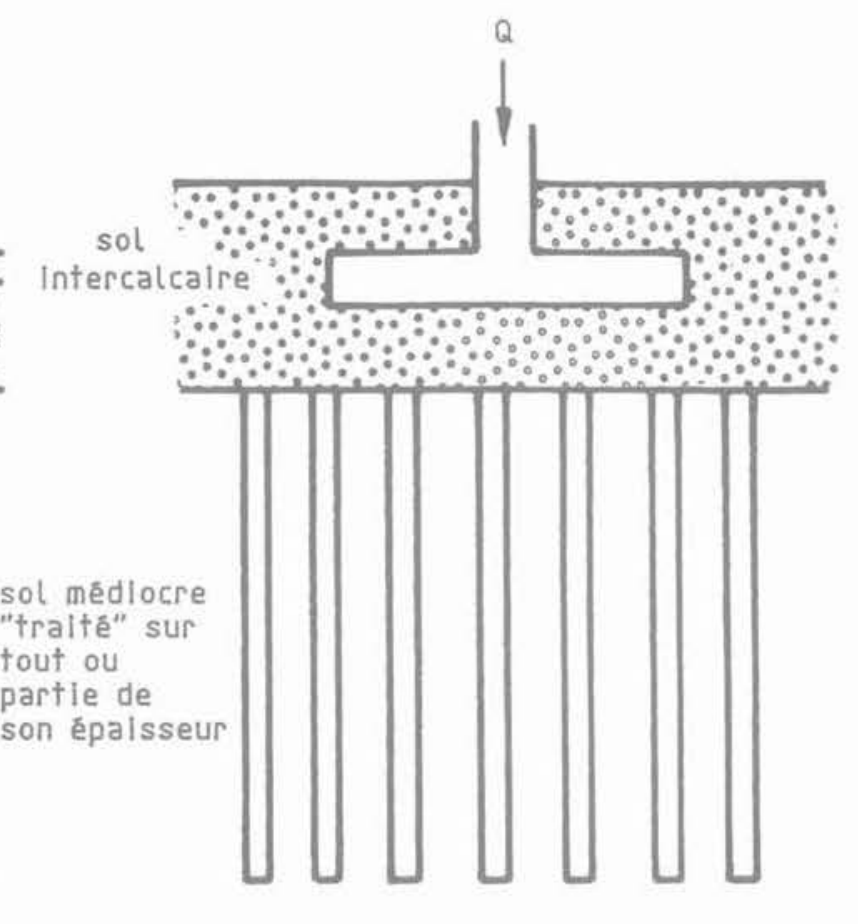

sol de bonne qualites a - radler b - semelle superficielle

Fig. 1. - Principe de conception.

Fig. 1. - Framing conception. 
rel lui-même qui fasse office de matelas intercalaire, le niveau de la tête des inclusions étant alors adapté à cet effet lors des travaux d'exécution.

Les caractéristiques mécaniques du sol intercalaire doivent, dans tous les cas, et cela est essentiel, être permanentes.

Pour les deux cas de figure ci-dessus, même sî les mécanismes généraux de fonctionnement sont, a priori, les mêmes, deux approches différentes sont proposées quant au dimensionnement du réseau.

La première s'applique à la configuration -a- et tient compte de la très grande dimension de l'ouvrage, qui met en jeu vis-à-vis du tassement du sol le phénomène de consolidation.

La seconde s'applique à la configuration -b- où la dimension restreinte de la fondation, alliée à sa rigidité, fait que sa justification peut relever des méthodes pressiométriques, auxquelles on fera effectivement appel.

\section{RADIER INFINIMENT SOUPLE}

Pour le cas idéal où la fondation est de très grande dimension et infiniment souple, on peut dimensionner le réseau de renforcement en appliquant, tout en les adaptant, les principes utilisés dans le cas du remblai construit suivant ce procédé ; le lecteur se reportera aux travaux antérieurs déjà cités en introduction, pour les développements théoriques. Le but recherché est bien sûr le mêrne que pour le remblai, c'està-dire une diminution des déformations, conduisant en surface du sol à une réduction importante des tas- sements verticaux de la structure : on fait volontairement abstraction des effets de bord.

La figure 2 récapitule lensemble des paramètres intervenant dans le calcul, avec le sol médiocre supposé homogène par souci de simplification.

En faisant l'hypothèse que l'effort en tête d'une inclusion est transmis par cisaillement le long d'une colonne fictive, prolongeant l'inclusion dans l'épaisseur $h_{\mathrm{r}}$ de la couche intercalaire, caractérisée par son angle de frottement $\varphi_{\mathrm{r}}$, on démontre que la contrainte résiduelle sur le sol à la base du matelas vaut:

$$
q^{\prime}\left(h_{r}\right)=p e^{-m_{r} h_{r}}+\frac{\gamma_{t}}{m_{r}}\left(1-e^{-m_{r} h_{r}}\right)
$$

dans laquelle, le coefficient $\mathrm{m}_{\mathrm{r}}$ a pour expression (K traduisant le "serrage » du matériau), avec un sol très très frottant:

$$
\mathrm{m}_{\mathrm{r}}=\frac{2 \mathrm{~K} \operatorname{tg} \varphi_{\mathrm{r}}}{\mathrm{R}\left(\frac{\mathrm{b}^{2}}{\mathrm{R}_{2}}-1\right)}
$$

On peut d'ailleurs améliorer de manière importante l'efficacité du matelas de répartition en conférant à celui-ci une cohésion $C_{r}$ qui favorise le transfert des efforts vers la tête des inclusions. On montre, en effet, que la contrainte résiduelle est alors donnée par :

$$
\begin{aligned}
& q^{\prime}\left(h_{r}\right)=p e^{-m_{r} h_{r}}+ \\
& \left(\frac{\gamma_{r}}{m_{r}}-\frac{C_{r}}{K \operatorname{tg} \varphi_{r}}\right)\left(1-e^{-m_{r} h_{r}}\right)
\end{aligned}
$$

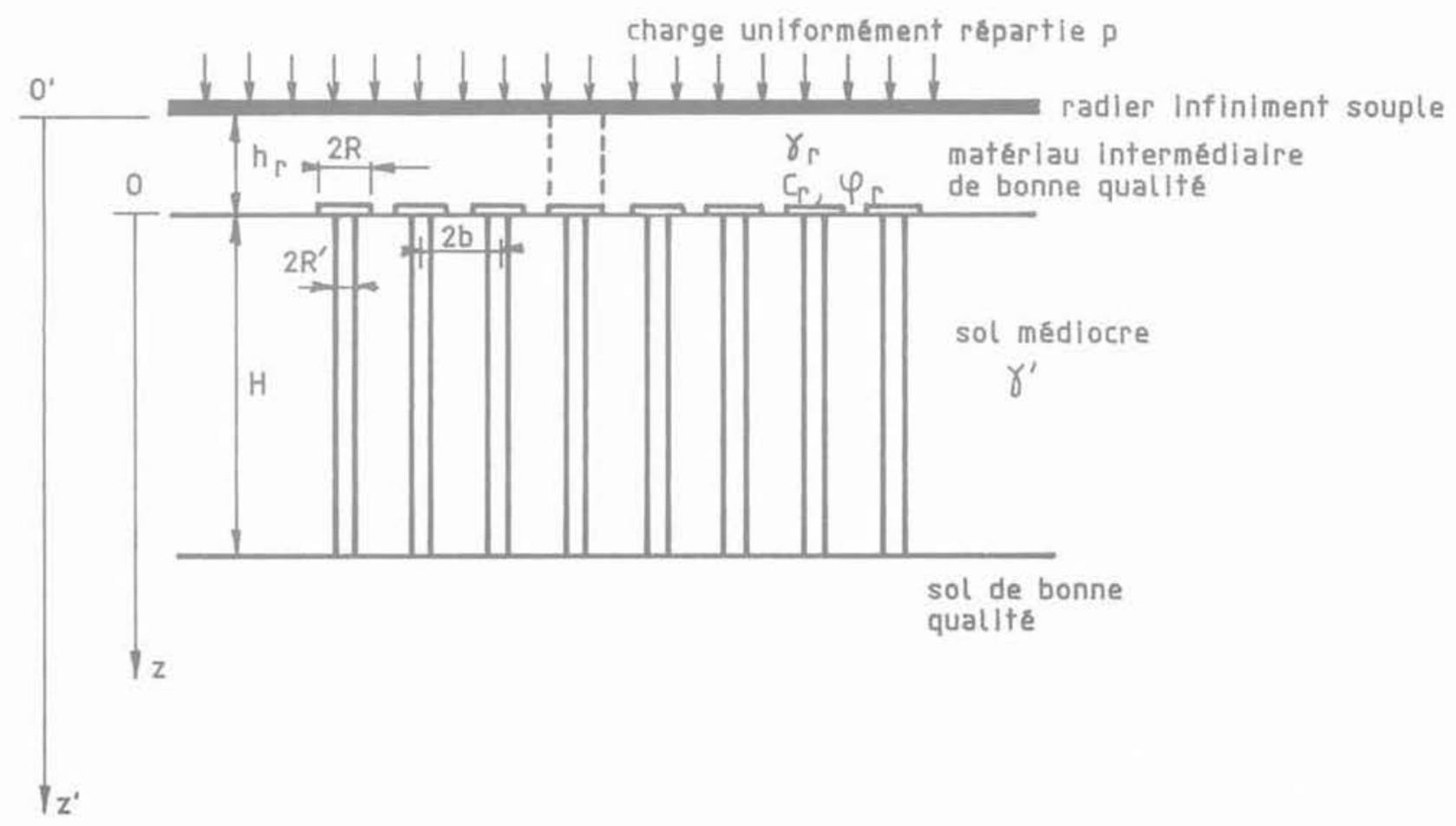

Fig. 2. - Principe de construction d'un radier.

Fig. 2. - Working principle of flexible raft foundation. 
Ainsi l'utilisation d'un matériau tel que le Texsol, mélange intime de sable et de fil continu, doué d'une cohésion verticale $\mathrm{C}_{\mathrm{r}}$, ou bien l'incorporation de nappes de géotextiles conférant au matériau l'équivalent d'une cohésion, s'avèrent-elles, a priori, très intéressantes.

On retrouve bien dans ces formules, pour $p=0$, l'expression applicable au remblai seul reposant sur le réseau, et pour $h_{r}=0$, la charge $p$, uniforme par définition, chargeant directement la surface du sol et les inclusions.

La validité de l'expression (1) repose sur les mêmes hypothèses que dans le cas de la construction d'un remblai, à savoir une déformation relative verticale suffisante entre la surface du sol et les têtes d'inclusions, indispensable à la mobilisation de l'effet de voûte au sein du matelas intercalaire; ce sera toujours le cas dans les situations où le projeteur concevra une telle solution de fondations.

La connaissance de $q^{\prime}\left(h_{\mathrm{r}}\right)$ et du maillage défini par $\mathrm{b} / \mathrm{R}$ permet le calcul de l'effort transmis en tête d'inclusion ; s'ajoute à cet effort, celui produit par frottement négatif le long du fût, au sein des sols médiocres, sous la contrainte $\mathrm{q}^{\prime}\left(\mathrm{h}_{\mathrm{r}}\right)$, dont la connaissance permet de dimensionner le fût de l'inclusion à laquelle on pourra donner un rayon R' inférieur au rayon $\mathrm{R}$ de la tête; le calcul des contraintes verticales dans le sol induites par $q^{\prime}\left(h_{r}\right)$ et réduites en profondeur par suite du frottement négatif permet d'estimer le tassement final de la structure.

Comme le montrent les exemples numériques qui suivent, il apparaît à l'évidence, étant donné l'épaisseur de $h_{r}$ généralement modérée qui sera donnée au matelas, que pour réduire les tassements différentiels et distorsions qui ne manqueraient pas de se produire entre la surface du sol médiocre et les têtes d'inclusions si celles-ci étaient fixes, il est indispensable que l'effort supporté par chacune d'elle soit proche de sa capacité portante ultime : cette condition permet un tassement suffisant, mais qui reste néanmoins toujours inférieur à celui du sol environnant sous la contrainte $\mathrm{q}^{\prime}\left(\mathrm{h}_{\mathrm{r}}\right)$; on devra donc favoriser un tassement d'ensemble avec, par inclusion, une charge transmise supérieure à sa charge critique de fluage.

\section{Exemple numérique}

Il concerne un réservoir de très grand diamètre, reposant sur $10 \mathrm{~m}$ de sol compressible par l'intermédiaire d'un matelas sablo-graveleux compact. Les principales données sont les suivantes:

$-p:$ (stockage liquide) $=100 \mathrm{kN} / \mathrm{m}^{2}$;

- matelas : $\mathrm{h}_{\mathrm{r}}=1,00 \mathrm{~m} ; \gamma_{\mathrm{r}}=20 \mathrm{kN} / \mathrm{m}^{3}$;

$\mathrm{K} \operatorname{tg} \varphi_{\mathrm{r}}=1,00$ :

- sol compressible :

$$
\mathrm{H}=10 \mathrm{~m} ; \gamma^{\prime} \text { déjaugé }=10 \mathrm{kN} / \mathrm{m}^{3} \text {; }
$$$$
\mathrm{K} \operatorname{tg} \delta=0,15 ; \frac{\mathrm{C}_{\mathrm{c}}}{1+\mathrm{e}_{\mathrm{o}}}=0,15
$$

- réseau d'inclusion, à maille carrée :

$$
\mathrm{R}=0,30 \mathrm{~m} ; \frac{\mathrm{b}^{2}}{\mathrm{R}_{2}}=7
$$

Remblai et cuve apportent au sol non traité la contrainte $\mathrm{q}=\mathrm{p}+\gamma_{\mathrm{r}} \mathrm{h}_{\mathrm{r}}=120 \mathrm{kPa}$ d'où un tassement, sans amélioration du sol, qui atteindrait :

$$
\begin{gathered}
W=0,435 \mathrm{H} \frac{\mathrm{C}_{\mathrm{c}}}{1+\mathrm{e}_{\mathrm{o}}} \\
{\left[\frac{\mathrm{q}}{\gamma^{\prime} \mathrm{H}} \operatorname{Ln}\left(1+\frac{\gamma^{\prime} \mathrm{H}}{\mathrm{q}}\right)+\operatorname{Ln}\left(1+\frac{\mathrm{q}}{\gamma^{\prime} \mathrm{H}}\right)\right]=1 \mathrm{~m}^{.}}
\end{gathered}
$$

Le sol traité par les inclusions, telles que définies cidessus, avec :

$$
\mathrm{m}_{\tau}=\frac{2 \mathrm{~K} \operatorname{tg} \varphi_{\mathrm{r}}}{\mathrm{R}\left(\frac{\mathrm{b}^{2}}{\mathrm{R}_{2}}-1\right)}=1,111
$$

conduit en surface du sol naturel à :

$$
\begin{aligned}
\mathrm{q}^{\prime}\left(h_{\mathrm{r}}\right) & =100 \mathrm{e}^{-1.111} \\
& +\frac{20}{1,111}\left(1-\mathrm{e}^{-1.111}\right)=50 \mathrm{kPa}
\end{aligned}
$$

Sous cette contrainte résiduelle de $50 \mathrm{kPa}$ à la surface du sol mou, la prise en compte du frottement négatif le long des inclusions au sein du sol compressible décharge ce dernier. Le calcul des contraintes verticales dans le sol montre que le frottement négatif agit sur $4,20 \mathrm{~m}$. Le calcul du tassement conduit à $42 \mathrm{~cm}$; le tassement de $1 \mathrm{~m}$, sans traitement, est donc ainsi divisé par 2,38. L'effort calculé dans l'inclusion atteint $222 \mathrm{kN}$, ce qui intrinsèquement faible et nécessiterait une adaptation du diamètre du fût courant de l'inclusion.

Si l'on dispose un matelas intercalaire de $2 \mathrm{~m}$ d'épaisseur, avec le même réseau d'inclusions, on vérifie que le tassement, de $1.06 \mathrm{~m}$ avec tout traitement, est ramené à $28 \mathrm{~cm}$, soit un rapport de réduction de 3,78 , la contrainte résiduelle étant alors de $30 \mathrm{kPa}$.

Dans ce même exemple où l'épaisseur $h_{\mathrm{g}}$ du matelas est de $1 \mathrm{~m}$, si celui-ci possède une cohésion de $50 \mathrm{kPa}$, la contrainte q'(hr) est alors réduite à $17 \mathrm{kPa}$ et le tassement final atteint $20 \mathrm{~cm}$.

Dans les trois cas, la charge par inclusion atteignant donc environ $230 \mathrm{kN}$, le dimensionnement de celleci sera tel que cette valeur soit comprise entre la charge critique et la charge limite de linclusion. On assurera de la sorte à la fois une réduction et une certaine homogénéité du tassement d'ensemble de la structure.

Si au cours de la vie de l'ouvrage, on est amené à augmenter très substantiellement la charge qu'il supporte, de telle sorte que la charge limite des inclusions soit totalement mobilisée voire dépassée, c'est le sol seul en surface qui absorbera toutes les con.

\footnotetext{
- Cette formule, peu usitée, est l'expression exacte du tassement qui diffère très sensiblement de la formule simplifiée usuelle.
} 
traintes supplémentaires en résultant, entraînant bien sûr du tassement supplémentaire.

A tassement final égal souhaité, il existe donc diverses possibilités de dimensionnement en jouant sur le maillage du réseau, l'épaisseur du matelas intercalaire. et la capacité portante des inclusions.

Les constatations sur ouvrages, faites par des ingénieurs anglo-saxons ayant conçu la fondation de réservoirs importants par cette méthode d'inclusions, sont tout à fait conformes aux développements ci-dessus.

Tel qu'il a été décrit par THORNBURN (1983), l'exemple de la fondation de réservoirs apportant une charge $\mathrm{p}$ de $160 \mathrm{kPa}$, reposant sur un matelas graveleux de $2 \mathrm{~m}$ d'épaisseur, posé sur un réseau de pieux carrés $0,25 \mathrm{~m} \times 0,25 \mathrm{~m}$ espacés de $2 \mathrm{~m}$ et couverts de semelles individuelles carrées de $1 \times 1 \mathrm{~m}$, est particulièrement significatif. En outre, il a été noyé, lors de la mise en cuvre, au coeur du matelas sablograveleux, une dalle de béton qui lui confère une cohésion équivalente améliorant la répartition des efforts transmis aux têtes des pieux constituant les inclusions. On peut calculer, partant d'une contrainte à la surface du sol de $200 \mathrm{kPa}$, que le disposif mis en cuure ramène celle-ci à $25 \mathrm{kPa}$, ou à $15 \mathrm{kPa}$, si l'on admet que la dalle bétonnée équivaut à donner au matelas intercalaire une cohésion de $10 \mathrm{kPa}$.

La réduction de contrainte a été effectivement très forte comme l'indiquent les faibles tassements mesurés sur site compris entre 30 et $50 \mathrm{~mm}$, le sol naturel possédant des caractéristiques de compressibilité élevée; de même, la charge calculée transmise aux inclusions représente $90 \%$ de la charge totale, en très bon accord avec les constatations relevées et les prévisions des auteurs.

Toutes les applications de fondations par cette méthode d'amélioration des sols médiocres avec des inclusions rigides, prouvent l'efficacité du procédé, avec des résultats remarquables quant à la réduction des tassements (ABBS, 1984; RANDOLPH, 1983).

\section{SEMELLE RIGIDE DE FAIBLE DIMENSION}

Ce second cas concerne les semelles rigides de dimension transversale faible, d'usage courant, et dont le dimensionnement et le comportement peuvent être abordés par les méthodes pressiométriques.

Le mécanisme de fonctionnement est le même que précédemment. Par l'intermédiaire du sol intercalaire, la semelle transmet une fraction de la charge appliquée, aux têtes d'inclusions: la charge résiduelle est directement transmise au sol environnant lequel, tassant plus que la partie haute des inclusions, soumet celles-ci à des efforts de frottement négatif sur une hauteur $h_{c}$. A cette profondeur $h_{c}$, variable suivant la position transversale de linclusion sous la semelle, par suite des effets de bord, l'effort dans l'inclusion $Q_{1}+$ $F_{n}$ est maximal. Cet effort est partiellement retransmis par frottement positif $F_{p}$ au-delà de $h_{c}$, et par $Q_{p}$ en pointe (fig. 3).

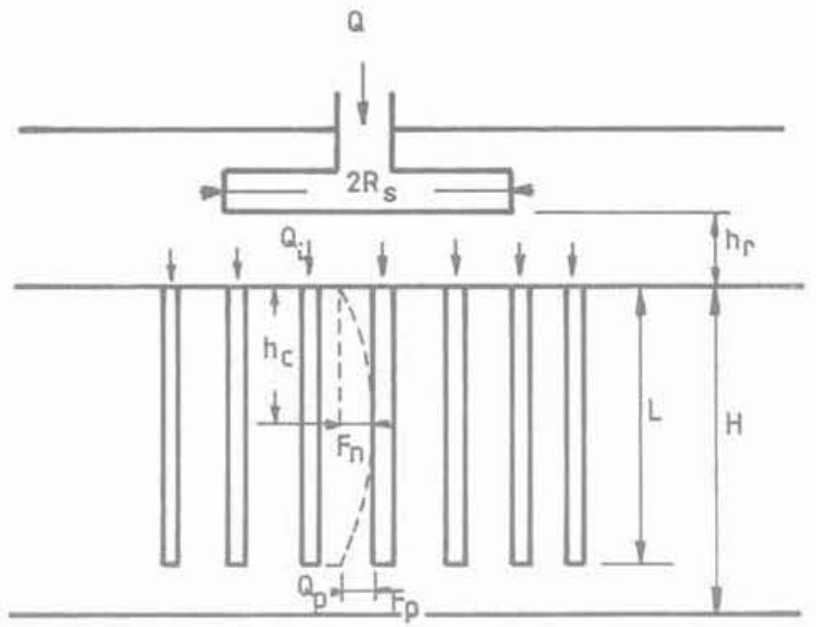

Fig. 3. - Mécanisme de fonctionnement sous une semelle rigide.

Fig 3. - Working principle of rigid tooting

A cette répartition des efforts résultant de la seule action Q sur la semelle, se superpose une répartition de contraintes due au poids propre du sol intercalaire, qui est généralement un remblai d'apport. Son épaisseur, volontairement modérée entre base de la semelle et têtes d'inclusions, fait que cette sollicitation complémentaire reste faible, sans commune mesure avec celle résultant de la charge $\mathrm{Q}$.

Le traitement du sol par les inclusions rigides a deux conséquences sur le comportement de la semelle :

- pour une charge appliquée $\mathrm{Q}$ donnée, le tassement est moindre que si le sol n'était pas renforcé. C'est le principal objectif du renforcement ;

- la capacité portante (ou charge limite) de la fondation est améliorée, puisque l'effort appliqué est partiellement transmis par les inclusions à des couches profondes du sol hors de la zone d'influence de la semelle.

Cette capacité d'amélioration dépend de la possibilité et de la capacité de transfert des efforts par chacune des inclusions. Les deux facteurs prépondérants dans celles-ci sont la capacité portante en pointe de linclusion, qui limite l'effort qu'elle peut supporter en tête. et la distance $h_{\mathrm{r}}$ entre cette tête et la base de la semelle, qui influence la dissipation des contraintes sous celle-ci. Si $h_{r}$ devient supérieur à une dimension dont on peut fixer la limite de 1 à 1.5 fois la largeur $2 \mathrm{R}_{5}$ de la semelle, on peut dire que, vis-à-vis de la capacité portante, l'efficacité du réseau devient pratiquement nulle.

Etant donné les difficultés, liées à la faible dimension de la largeur de la semelle, il est proposé un modèle de dimensionnement relativement simple qui respecte des solutions connues pour certains cas de figures particuliers.

\section{Principe de justification}

La figure 4 rassemble 6 schémas de principe correspondant à autant de cas de figure de fondations susceptibles d'être mis en œuvre, dont certains correspondent à des dispositions extrêmes d'inclusions de longueur nulle, ou en contact avec la semelle. 


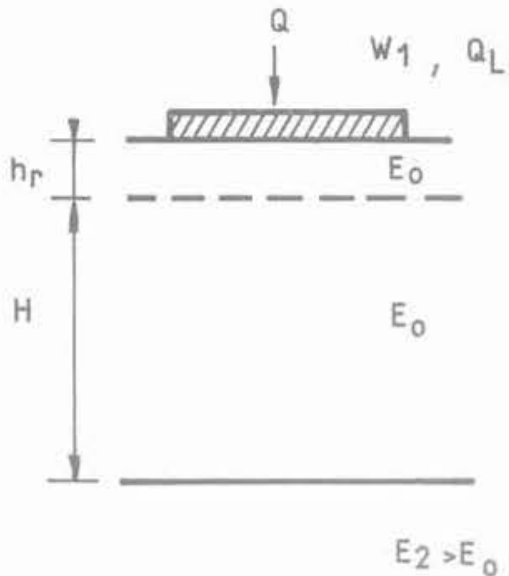

(1)

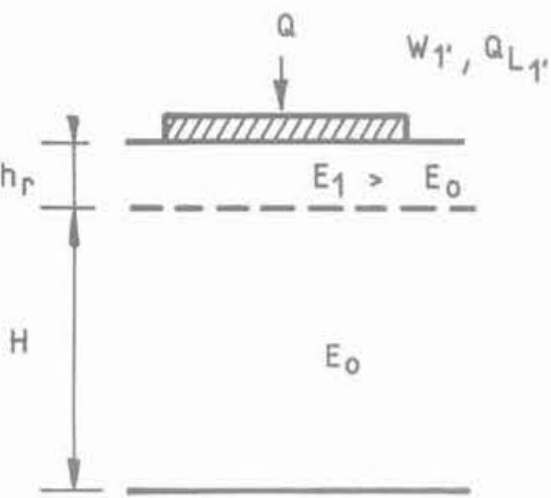

E2

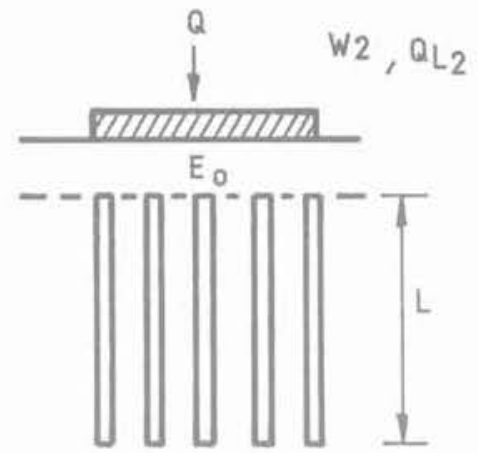

$E_{2}$
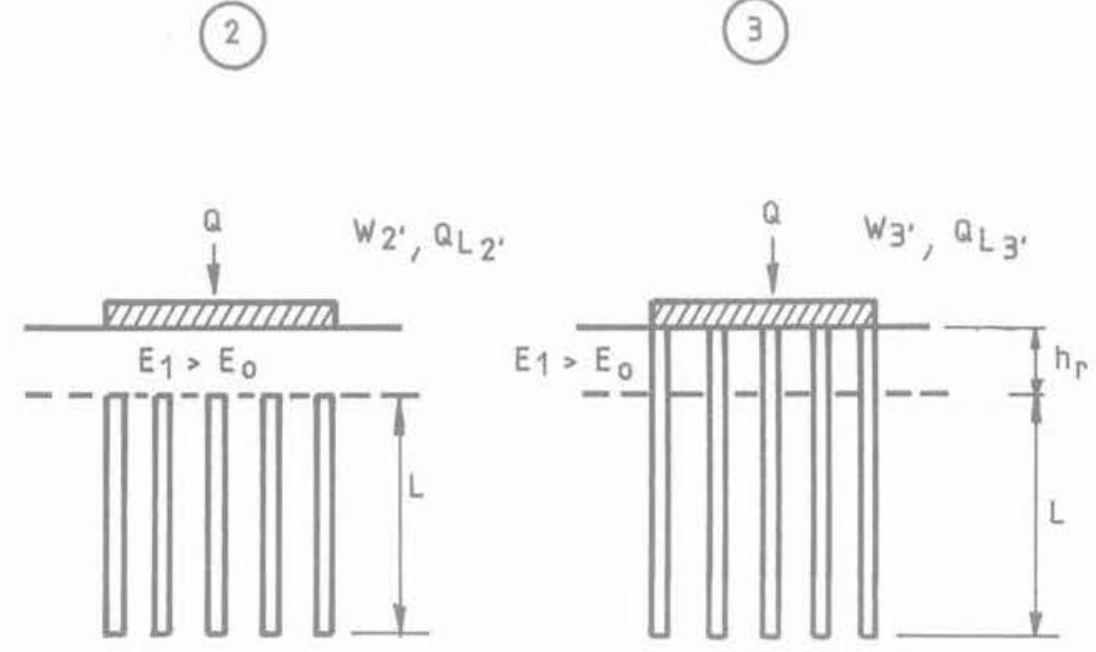
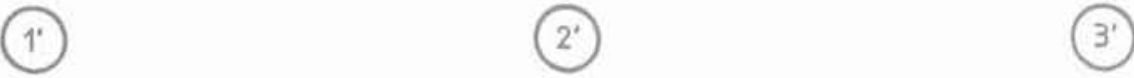

Fig. 4. - Configuration de fondations.

Fig. 4. - Foundations types.

Les géométries sont identiques, la seule variable étant la déformabilité du matelas intercalaire d'épaisseur $h_{r}$. constitué soit de sol naturel, de module de déformation pressiométrique $\mathrm{E}_{\mathrm{o}}$, soit d'un sol de meilleure qualité, de module $E_{1}>E_{0}$. Les paramètres $H, L$, $E_{2}, E_{0}$ sont fixés. On aura, en outre, le plus souvent $\mathrm{H}>\mathrm{L}>\mathrm{h}_{\mathrm{r}}$.

On peut, pour ces six schémas de principe, hiérarchiser les valeurs du tassement $W$ obtenu sous charge imposée $\mathrm{Q}$, et les valeurs des charges limites $\mathrm{Q}_{\mathrm{L}}$, ce que traduisent les diagrammes de la page suivante.

On peut considérer, qu'en l'état actuel des connaissances, les tassements et charges limites sont calculables pour les situations 1, 3, 1', 3', en s'aidant des méthodes pressiométriques, à partir des caractéristiques de déformabilité et de résistance du sol (module $\mathrm{E}$, pression limite $\left.\mathrm{P}_{1}\right)$. Pour 1 et 1 ', il s'agit de fon- dations superficielles, pour 3 et 3 ' de fondations mixtes semelle-pieux.

Pour les situations 2 et 2', le paramètre important, influant sur la valeur du tassement $W$ ou de la charge $Q_{L}$, est le rapport $h_{r} / R_{s}$ de l'épaisseur du matelas intercalaire à la demi-largeur de la semelle.

Pour la capacité portante, en se référant à la théorie pressiométrique, si $h_{\text {r }}$ excède 2 à $3 \mathrm{R}_{\mathrm{s}}$, le sol sousjacent au matelas, qu'il soit amélioré ou non, n'a plus d'influence sur la charge limite. Seule la qualité du matelas joue un rôle. Les théories plus classiques des bicouches le montrent également.

Pour les tassements, selon la même théorie, linfluence

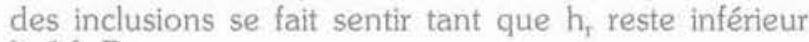
à $16 \mathrm{R}_{\mathrm{s}}$. 

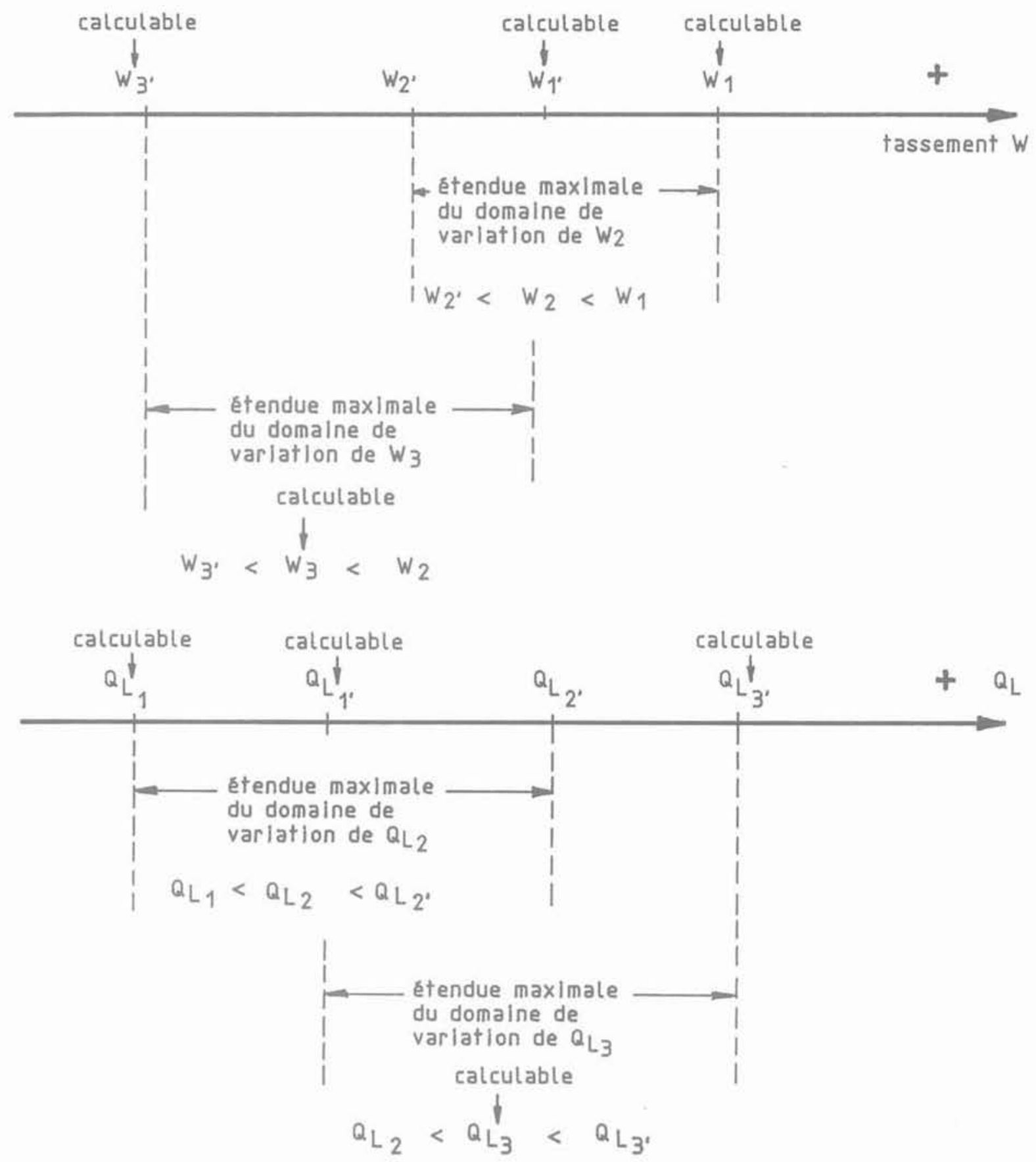

Les figures 5 (page suivante) donnent lallure des variations de $Q_{L}$ et $W$ (cas d'une configuration ou $H$ $\gg R_{\mathrm{S}}$.

On convient d'adopter les expressions suivantes donnant les charges limites:

$$
\begin{aligned}
& \mathrm{Q}_{\mathrm{L}_{2}}=\mathrm{Q}_{\mathrm{L}_{1}} \text { si } \mathrm{h}_{\mathrm{r}}>2 \mathrm{R}_{\mathrm{s}} \\
&\left.\mathrm{Q}_{\mathrm{L}_{2}}=\mathrm{Q}_{\mathrm{L}_{1}}+\frac{1}{4}\left(2-\frac{\mathrm{h}_{\mathrm{r}}}{\mathrm{R}_{\mathrm{s}}}\right)^{2} \mathrm{Q}_{\mathrm{L}_{3}}-\mathrm{Q}_{\mathrm{L}_{1}}\right) \\
& \text { si } \mathrm{h}_{\mathrm{r}}<2 \mathrm{R}_{\mathrm{s}}
\end{aligned}
$$

$$
\begin{array}{lr}
\mathrm{Q}_{\mathrm{L}_{2}}=\mathrm{Q}_{\mathrm{L}_{1}} & \text { si } \mathrm{h}_{\mathrm{r}}>2 \mathrm{R}_{\mathrm{s}} \\
\mathrm{Q}_{\mathrm{L}_{2}}=\mathrm{Q}_{\mathrm{L}^{\prime}}+\frac{1}{4}\left(2-\frac{\mathrm{h}_{\mathrm{r}}}{\mathrm{R}_{\mathrm{s}}}\right)^{2}\left(\mathrm{Q}_{\mathrm{L}^{\prime}}-\mathrm{Q}_{\mathrm{L}^{\prime}}\right)
\end{array}
$$

ou bien :

$$
\mathrm{Q}_{\mathrm{L}_{2}}=\mathrm{Q}_{\mathrm{L}_{2}}+\mathrm{Q}_{\mathrm{L}^{\prime}}-\mathrm{Q}_{\mathrm{L}_{1}} \quad \text { si } \mathrm{h}_{\mathrm{r}}<2 \mathrm{R}_{\mathrm{s}}
$$

ces deux dernières égalités étant équivalentes, puisque l'on peut supposer que l'on aura toujours pratiquement $\mathrm{Q}_{\mathrm{L}_{3}}-\mathrm{Q}_{\mathrm{L}_{1}}=\mathrm{Q}_{\mathrm{L}_{3}}-\mathrm{Q}_{\mathrm{L}_{1}}$, étant donné les dimensions relatives pratiques des différentes grandeurs $R_{s}, h_{r}, L, H$. 


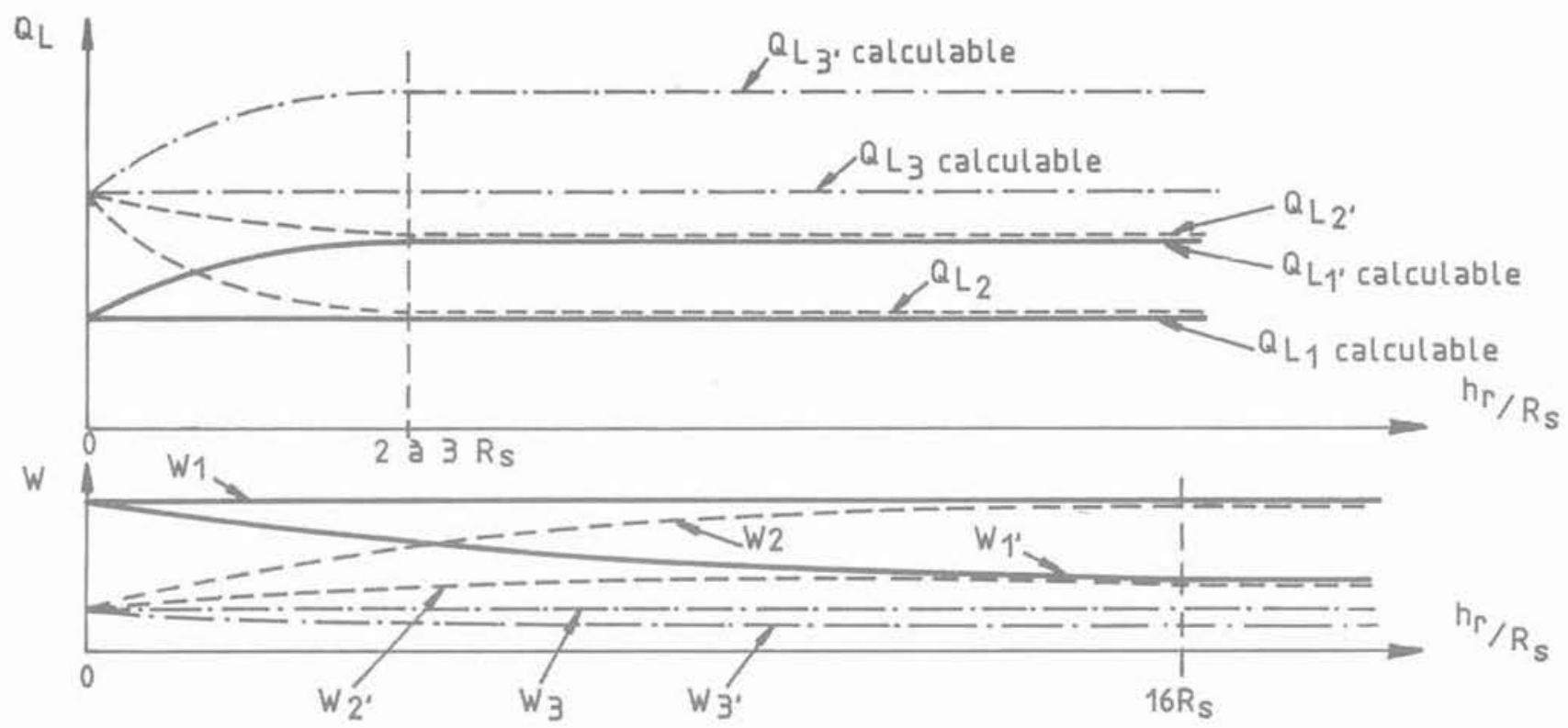

Fig. 5. - Variation des charges limites et tassement avec $h_{r}$

Fig. 5. - Relation between ultimate loads, settlements and depth $h$, of fill material.

Dans ces expressions, on a volontairement choisi une valeur limite basse, de 2 , pour $h_{f} / R_{s}$, qui constitue ainsi une sécurité pour le calcul de $\mathrm{Q}_{\mathrm{L}_{2}}$ ou $\mathrm{Q}_{\mathrm{L}_{2}}$, et une représentation de type parabolique, assurant une continuité, a priori, logique de la dérivée $\mathrm{dQ}_{\mathrm{L}} / \mathrm{dh}_{\mathrm{r}}$, pour $h_{\mathrm{r}}=2 \mathrm{R}_{\mathrm{s}}$

Le calcul de $\mathrm{Q}_{\mathrm{L}_{2}}$ ou $\mathrm{Q}_{\mathrm{L}_{2}}$, nécessite donc le calcul préalable des charges limites $\mathrm{Q}_{\mathrm{L}_{3}}, \mathrm{Q}_{\mathrm{L}_{3}}, \mathrm{Q}_{\mathrm{L}_{1}}, \mathrm{Q}_{\mathrm{L}_{1}}$.

Ces expressions admettent implicitement l'hypothèse qu'il n'y a pas d'influence réciproque entre les différentes composantes de la charge limite. Il faut noter que la réalité en diffère légèrement, puisque les inclusions interceptent les lignes de moindre résistance au cisaillement se développant sous la semelle, ce qui est donc négligé, de même que l'on peut évoquer l'aug. mentation du * confinement " du sol autour des inclusions (du fait des pressions transmises par la semelle), qui peut tendre à accroitre légèrement la capacité portante de celles-ci. Le terme supplémentaire de $\alpha$ mutuelle influence » qui en résulterait est donc négligé : il est néanmoins probablement petit.

Les formules (1) et (1'), outre le rôle important des termes $\left(Q_{L_{3}}-Q_{L_{1}}\right)$, capacité portante propre au réseau, et $\left(\mathrm{Q}_{\mathrm{L}_{1}}-\mathrm{Q}_{\mathrm{L}_{1}}\right)$, capacité portante additive due au matelas $h_{r}$, montrent que cette épaisseur $h_{\text {r }}$ devra être faible, une bonne valeur pouvant raisonnablement être fixée au quart ou au tiers de $R_{\mathrm{s}}$. Trop épais, c'est uniquement la qualité propre du matelas qui confère un gain de capacité portante.

D'un point de vue pratique, il sera conseillé de prévoir, en fonction des dimensions respectives des éléments géométriques, la réalisaion d'inclusions en dehors de la stricte emprise géométrique de la semelle ; cette disposition, intuitive, sera adoptée, conformément au schéma ci-après, dès que $h_{r} \geq e$, espacement entre inclusions (fig. 6).

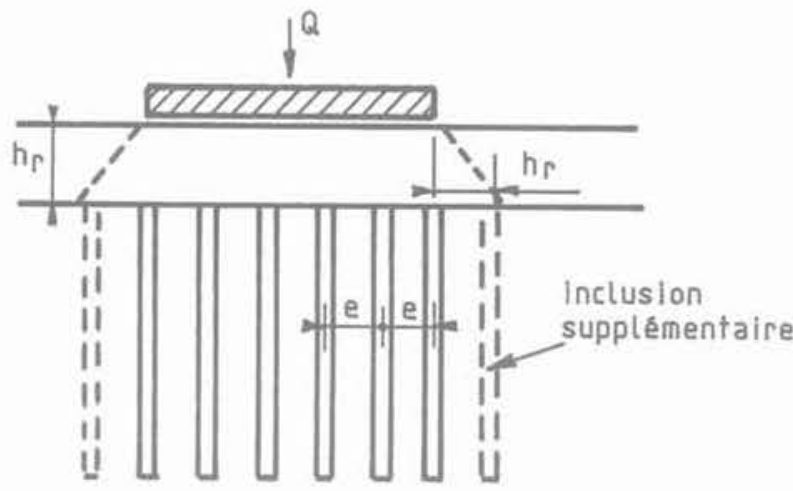

Fig. 6. - Disposition pratique d'un réseau d'inclusion. Fig. 6. - Practical arrangement of a rigid piles network.

On procèdera au calcul du tassement, en faisant appel à la notion de module pressiométrique équivalent. On détermine, dans le schéma 7a suivant représentant une fondation mixte sur le sol homogène $E_{o}$, la courbe complète effort-déformation par la méthode développée à cet effet (COMBARIEU, 1988).

On considère que cette courbe (7b) traduit le comportement d'une fondation superficielle, de charge limite $\mathrm{Q}_{L_{3}}$, de superficie identique à la semelle réelle, et reposant sur un sol stratifié, dont les caractéristiques de déformabilité de la couche d'épaisseur L sont celles d'un sol homogène possédant un module équivalent $\mathrm{E}_{\mathrm{o}, \mathrm{e}}(7 \mathrm{c})$.

On recherchera donc la valeur $E_{o e}$ qui rende le mieux compte, dans le cas de la fondation superficielle équivalente, de la courbe charge-tassement de la fondation mixte.

Le calcul final de la semelle sur sol amélioré (cas 2') peut ensuite s'effectuer, suivant le schéma équivalent ci-dessous (fig. 8). 


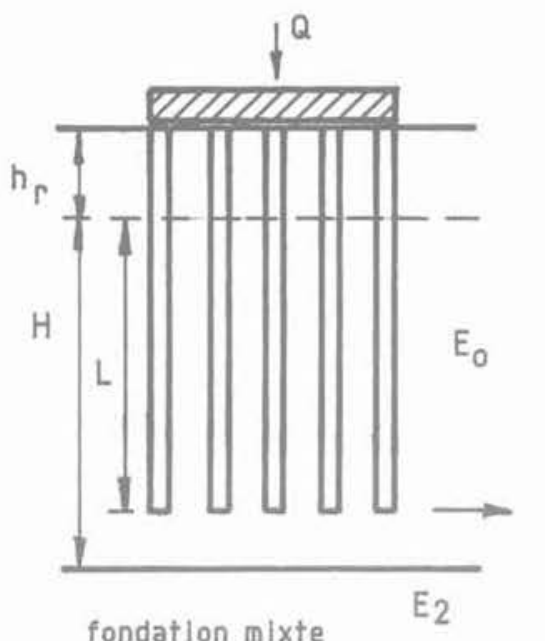

(a)

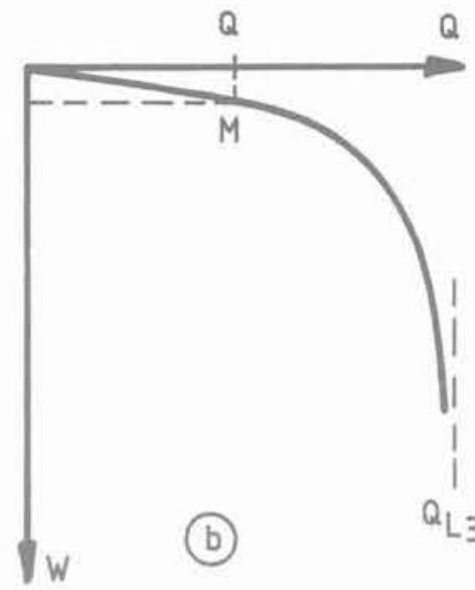

Q

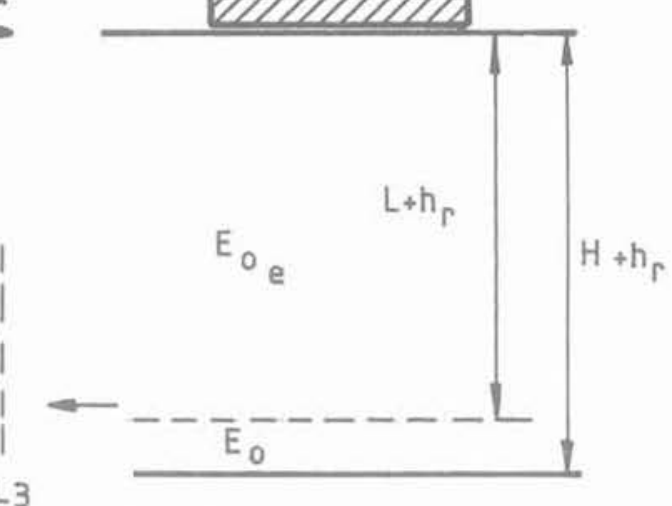

fondation superficlelle equivalente

Fig. 7. - Principe du module équivalent.

Fig. 7. - Equivalent modulus principle

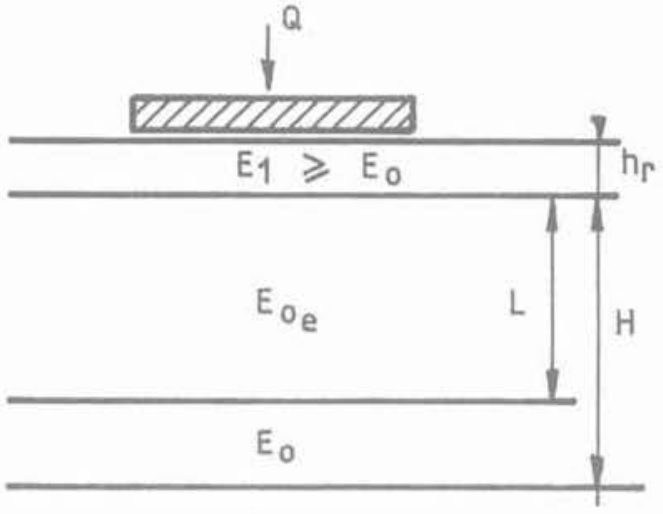

$E_{2}$

Fig. 8. - Calcul de la semelle avec module équivalent.

Fig. 8. - Design of footing with equivalent modulus.

\section{Essais en station du CEBTP}

Ces essais, dont les résultats détaillés ont été publiés par PLUMELLE (1985), ont consisté dans le chargement d'une semelle reposant par l'intermédiaire d'un matelas de matériau, sur un massif de sable peu compact, amélioré par des micropieux $\varnothing 84 \mathrm{~mm}$.

Le matelas a été successivement du sable, analogue à celui du massif, puis de la grave beaucoup plus compacte.

L'ensemble des essais, auxquels on se reportera, permet une analyse intéressante au regard du modèle de dimensionnement proposé.

Essai direct de la semelle sur massif de sable seul (semelle $1 \mathrm{~m} \times 1 \mathrm{~m}$ )

Si on se place au taux d'enfoncement de $5 \%$ du diamètre, la charge limite mesurée $\mathrm{Q}_{\mathrm{L}_{1}}$ vaut $270 \mathrm{kN}$.
Essai de semelle, avec matelas intercalaire de $50 \mathrm{~cm}$ de sable, et sable amélioré par inclusions

La géométrie des inclusions fait que 4 inclusions intéressent directement la semelle; ces 4 inclusions ont une charge limite, en groupe (un essai de chargement direct du groupe le prouve) de $200 \mathrm{kN}$, essentiellement due au frottement. La fondation mixte composée de la semelle et de quatre pieux de longueur $6 \mathrm{~m}$ qui est la longueur des inclusions, a donc une charge portante limite $Q_{L_{3}}$ de $270+11 / 12 \times 200$ (on élimine le frottement sur une hauteur du fût des pieux de $50 \mathrm{~cm}=\mathrm{R}_{\mathrm{s}}$, demi-largeur de la semelle, conformément à la méthode de dimensionnement des fondations mixtes ce qui représente les $11 / 12$ de la longueur du fât), soit $\mathrm{Q}_{\mathrm{L}_{3}}=453 \mathrm{kN}$. La charge portante limite de la semelle sur sol renforcé, avec $h_{r} / R_{s}=1$, vaut donc:

$$
\begin{aligned}
Q_{L_{2}} & =Q_{L_{1}}+\frac{1}{4}\left(2-\frac{h_{t}}{R_{s}}\right)^{2}\left(Q_{L_{3}}-Q_{L_{1}}\right) \\
& =270+\frac{1}{4} \cdot 183=316 \mathrm{kN}
\end{aligned}
$$

Cette valeur est à comparer à la valeur de $310 \mathrm{kN}$ enregistrée lors de l'essai de chargement, pour une déformation de $50 \mathrm{~mm}$.

Si l'on examine les résultats en admettant que la semelle intéresse 9 micropieux, (disposition possible puisque ceux-ci sont distants de $0,50 \mathrm{~m}$, pour une semelle de $1 \mathrm{~m} \times 1 \mathrm{~m})$, cette charge limite $\mathrm{Q}_{\mathrm{L}_{3}}$ serait de :

$$
270+\frac{11}{12} \times 450=682 \mathrm{kN}
$$

donnant : $\mathrm{Q}_{\mathrm{L}_{2}}=270+\frac{1}{4} \times 412=373 \mathrm{kN}$. 


\section{Essai de semelle, avec tout-venant intercalaire de $50 \mathrm{~cm}$, et sable amélioré par inclusions}

Une inconnue subsiste pour cet essai, puisque l'on ne connaît pas la qualité mécanique du tout-venant. On choisira donc une gamme de valeurs (paraissant correctes au devant de sa compacité), de la pression limite du tout-venant de 3,4 ou 5 fois la pression limite du sable, laquelle est de $380 \mathrm{kPa}$.

Dans ces conditions, suivant la méthode pressiométrique, la pression limite équivalente $\mathrm{P}_{\text {le }}$ vaut successivement 550,660 et $650 \mathrm{kPa}$, donnant avec un facteur de portance $\mathrm{k}$ de 0,8 , la gamme de charges limites $Q_{L_{1}}$ de 440,480 et $520 \mathrm{kN}$.

La charge limite $\mathrm{Q}_{\mathrm{L}_{2}}$, de la semelle, vaut donc:

$\mathrm{Q}_{\mathrm{L}_{2}}=\mathrm{Q}_{\mathrm{L}_{2}}+\mathrm{Q}_{\mathrm{L}_{1}}-\mathrm{Q}_{\mathrm{L}_{1}}$, soit 486 à $566 \mathrm{kN}$, suivant la qualité de tout-venant pour 4 inclusions intéressant directement la semelle.

Si 9 inclusions sont concernées, la valeur de $\mathrm{Q}_{\mathrm{L}_{2}}$ varie de 543 à $623 \mathrm{kN}$.

On rapprochera ces valeurs du nombre de $530 \mathrm{kN}$, valeur mesurée pour $50 \mathrm{~mm}$ de tassement, la charge maximale ayant dépassé $650 \mathrm{kN}$ pour plus de $80 \mathrm{~mm}$ d'enfoncement.

PLUMELLE, dans son expérimentation, a équipé de jauges de déformation l'un des micropieux, pour lequel la répartition des efforts a été suivie en cours de chargement (fig. 9). On note, conformément aux prévisions que se développe en haut du fût, du frottement négatif, auquel succède le frottement positif. Mais ce frottement négatif se mobilise plus fortement, ce qui est normal, lorsque le matelas intercalaire est constitué de tout-venant. En effet, si pour les deux cas de figure, on impose une même déformation verticale de la semelle, la charge correspondante au cas du tout-venant est supérieure à celle relative au sable. Le tassement vertical à la surface du sol initial en place est également supérieur dans le cas du toutvenant (fig. 10).

On en conclut, à tassement imposé, que la tête d'une inclusion est plus rapidement chargée, quand la couche intercalaire est meilleure, le frottement négatif étant également plus élevé : ceci est tout à fait conforme aux valeurs enregistrées lors de ces essais.

\section{Essais en centrifugeuse LPC}

BIGOT et CANEPA (1988) ont testé, à titre de faisabilité, des semelles de $1,5 \times 1,5 \mathrm{~m} ; 4,5 \mathrm{~m} \times 9 \mathrm{~m}$ et $9 \mathrm{~m} \times 18 \mathrm{~m}$, reposant par l'intermédiaire d'un matelas sableux sur du sable de Labenne de poids volumique $16 \mathrm{kN} / \mathrm{m}^{3}$, amélioré par des inclusions ; le maillage est très large, l'espacement e entre inclusions atteignant suivant les essais, la demi-largeur ou la largeur de la semelle (fig. 11).

En accord avec les simulations de calcul que l'on peut faire sur les essais réalisés, il a été mis en évidence

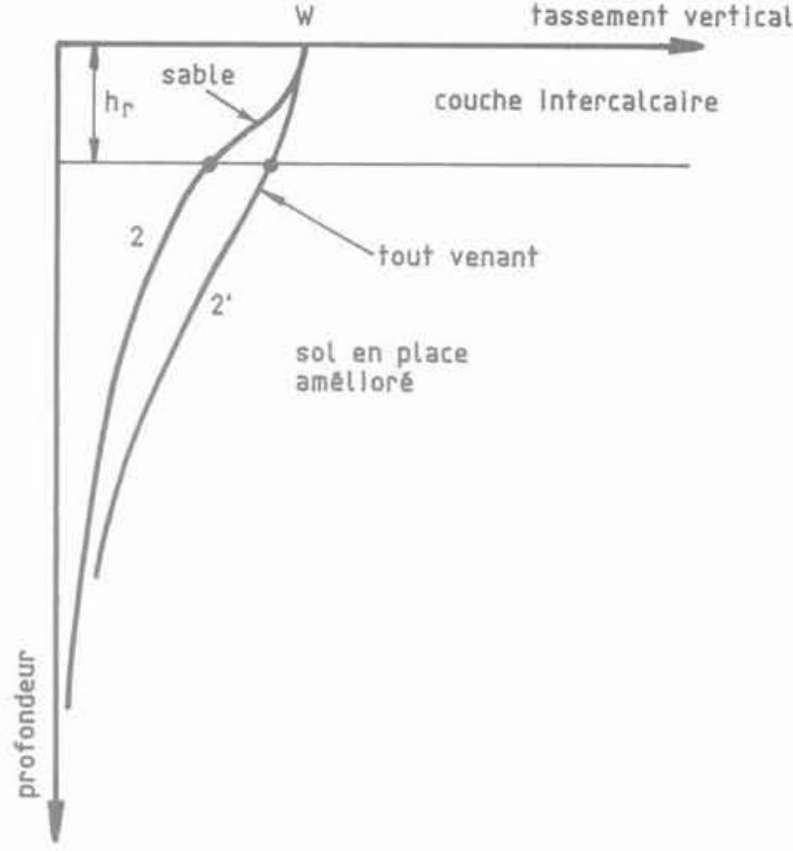

Fig. 10. - Tassement du sol en profondeur, suivant la qualité de la couche intercalcaire.

Fig 10. - Settlement of soil with depth. according to stiffness of fill material.

une efficacité quasi-nulle des réseaux d'inclusions étant donné : $1^{\text {er }}$ leur maillage très faible, $2^{e}$ leur charge intrinsèque faible.

\section{CONCLUSION}

L'amélioration de sols médiocres par inclusions rigides verticales s'avère efficace pour les radiers; la réduction de tassement peut être spectaculaire et l'efficacité du matelas intercalaire entre ouvrage et sol amélioré est d'autant plus grande que le matériau, qui doit posséder un bon angle de frottement interne, possède en outre une cohésion effective. Ces caractéristiques de frottement et cohésion doivent être permanentes.

On dimensionnera, en outre, les inclusions, de sorte qu'elles soient chargées au-delà de leur charge critique, voire près de leur charge limite.

Pour les semelles rigides, l'efficacité du procédé demande une charge portante intrinsèque des inclusions appréciables et une épaisseur de matelas intercalaire faible vis-à-vis de la largeur de la semelle. On cherchera d'ailleurs plus à réduire les tassements de la semelle, qu'à tenter d'augmenter la capacité portante, donc le taux de travail admissible. Dans ce dispositif, la qualité du matelas intercalaire lui-même a un rôle non négligeable dans cette réduction, qu'il est nécessaire, dans la justification du procédé, de bien dissocier du rôle propre joué par les inclusions. 

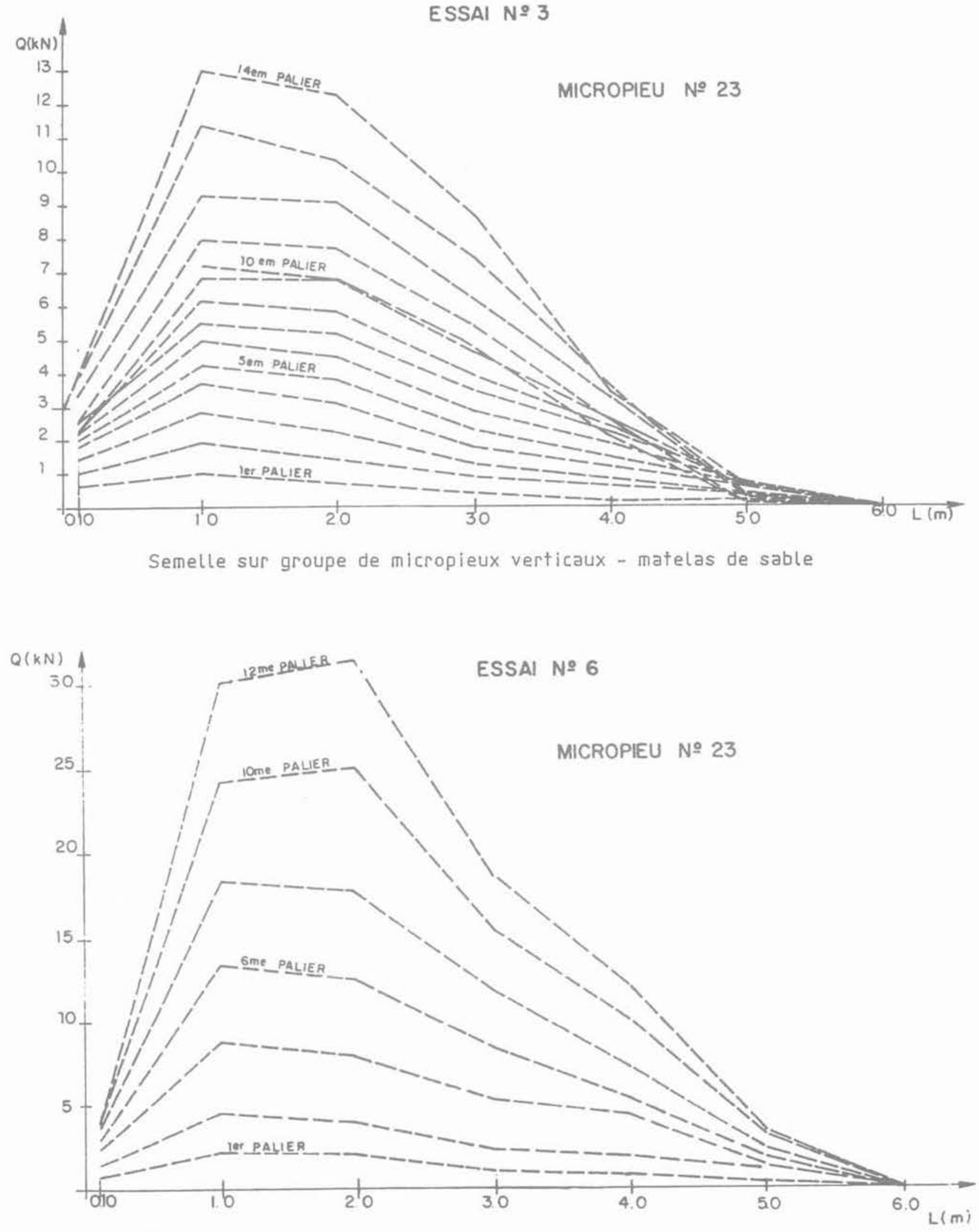

Semelle sur groupe de micropieux verticaux - matelas de tout venant

Fig. 9, - Répartition des efforts le long du fot. Essais de Plumelle.

Fig. 9. - Distribution of forces along the pile shaft. Load tests by Plumelle. 

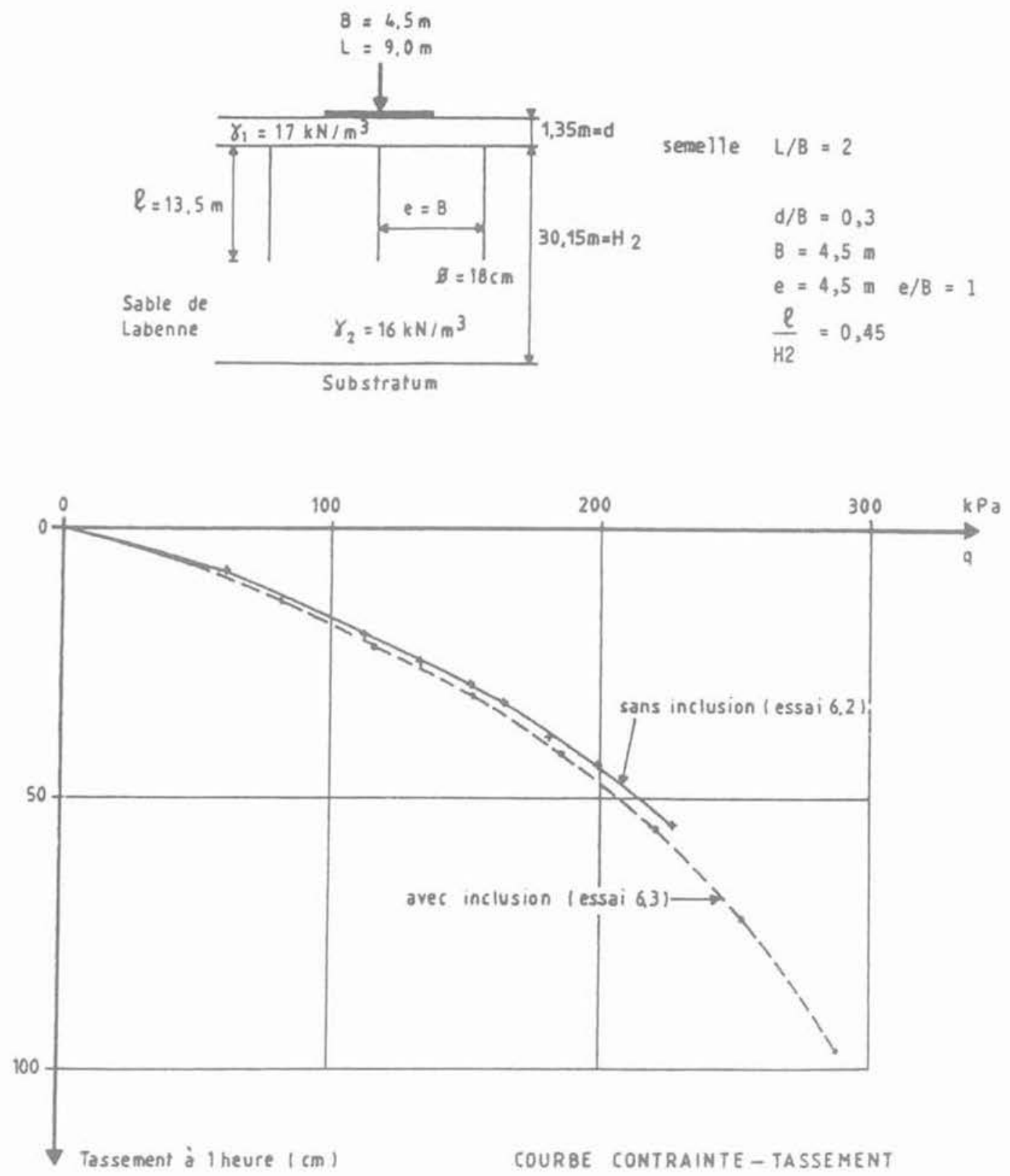

Fig. 11. - Essais (et notations) BIGOT-CANEPA sur semelle de 4,50 $\times 9,00 \mathrm{~m}$. Fig. 11. - Load test by BIGOT and CANEPA on $4.5 \times 9.00 \mathrm{~m}$ footing.

\section{BIBLIOGRAPHIE}

ABBS A.F. (1983), Le renforcement par pieux des sols de fondation d'un réservoir de pétrole. Colloque international sur le renforcement en place des sols et des roches, Paris, 1983, pp. 13 à 18.

BIGOT G., CANEPA Y. (1988), Fondation de bâtiments. Utilisation des techniques de traitement et d'amélioration des sols. Rapport interne LPC, pp. 37 à 41.

COMBARIEU O. (1987), Amélioration des sols par inclusions rigides verticales. Application à lédification des remblais sur sols médiocres. Rapport des Laboratoires LPC - GT 26. Revue Française de Géotechnique n 44, 1988, pp. 57 à 79.
COMBARIEU O. (1988), Calcul d'une fondation mixte semelle pieux sous charge verticale centrée. Note d'information technique LCPC, 1988, 15 p.

PLUMELLE C. (1985), Renforcement d'un sol lâche par inclusion de micropieux. Revue Française de Géotechnique n 30,1985 , pp. 47 à 57.

RANDOLPH M.F. (1983), Design of piled raft foundations. Recent developpements in laboratory and field tests and analyses of geotechnical problems. Bangkok, 1983, pp. 525 à 537.

THORBURN S., LAIRD C.L., RANDOLPH M.F. (1983), International conference on advances in piling and ground treatment. Institution of civils engineers. London, march 1983, pp. 157 à 164. 